\title{
Cognitive interference in prism adaptation
}

\author{
GORDON M. REDDING \\ Illinois State University, Normal, Illinois \\ and \\ BENJAMIN WALLACE \\ Cleveland State University, Cleveland, Ohio
}

\begin{abstract}
Visual and proprioceptive adaptation to optical displacement were found to decrease with increasing difficulty of mental arithmetic performed during hall exposure. The graded nature of such cognitive interference is consistent with the idea that limited-capacity central control processes are required to coordinate the discordant visual system with other sensorimotor systems. When central processing capacity is diverted to other tasks, the frequency of coordinated activity involving the discordant visual system is reduced, there are fewer occasions for the discordance to pose a problem, and there is less need for adaptive recalibration in any sensorimotor system.
\end{abstract}

Visual adaptation ${ }^{1}$ to optical distortion (displacement and tilt) is reduced by cognitive tasks (mental arithmetic and mental imagery) performed simultaneously with the exposure task of walking about hallways (Redding, Clark, \& Wallace, 1985). This interference cannot be attributed to any obvious decrease in exploratory behavior, because it persists even when experimental groups walk at the same rate or even faster than control groups not receiving the secondary task. To the extent that walking rate is an index of exploratory behavior and opportunity for perceptual learning, experimental groups should show the same or even greater adaptation than control groups. Nor can interference be attributed to preemption of perceptual mechanisms by the mental imagery component of the cognitive task (e.g., Segal \& Fusella, 1970), because its magnitude is unaffected by manipulations which can be assumed to vary this component. Requiring subjects to judge the angular difference between imaged hands for analog clock times does not produce greater interference than mental arithmetic, even though the clock task is at least as difficult as mental arithmetic and involves a larger mental imagery component. However, interference is not usually so great as to eliminate adaptation entirely, and the primary effect of secondary cognitive tasks seems to be to slow down rather than completely halt the adaptive process.

These observations are consistent with the idea that prism adaptation involves a limited-capacity cognitive mechanism (cf. Posner \& Snyder, 1975; Schneider \& Shiffrin, 1977) whose operation is necessary to establish

The research was supported in part by Grant 1-R03-MH34383 from the National Institute for Mental Health to the second author. Special thanks are due James Cummins for assistance in data collection. A preliminary report of these data was given to the 25th Annual Meeting of the Psychonomic Society, San Antonio, Texas, November 1984. Reprint requests should be addressed to Gordon M. Redding, Department of Psychology, Illinois State University, Normal, IL 61761. and maintain coordinative linkages between the discordant visual (eye-head) system and other sensorimotor systems (e.g., hand-head and ear-head). The directionality of coordinative linkages between discordant sensorimotor systems determines the locus of adaptation, with local recalibration occurring in the guided system rather than the guiding system (cf. Hamilton, 1964; Howard \& Templeton, 1966, p. 380). When central processing capacity is diverted to other tasks, the frequency of coordinated activity involving the discordant visual system is reduced, there are fewer occasions for the discordance to pose a problem and less need for adaptive recalibration in any sensorimotor system.

Figure 1 illustrates this directionality-of-guidance hypothesis (Redding et al., 1985) for the hall-exposure situation. Locomotion is assumed to be automatically guided by optical flow patterns (e.g., Fitch, Tuller, \& Turvey, 1982; Lee \& Thomson, 1982) that are not distorted by the prism. ${ }^{2}$ Consequently, walking during

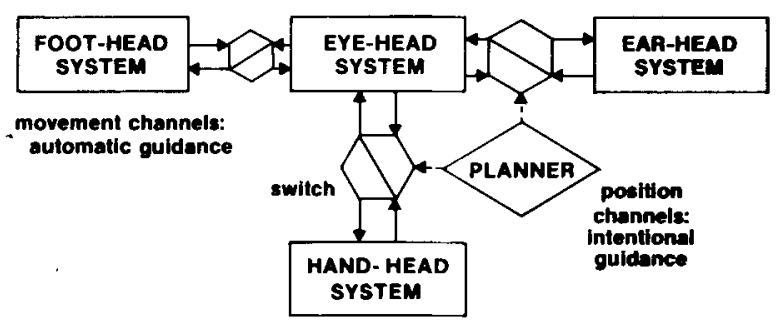

Figure 1. Directionality of guidance model for hall exposure. Locomotion is automatically guided by undistorted optical flow, whereas intentional guidance based on distorted positional information is subject to interference from cognitive tasks with consequentially less adaptation. Spatial information enters this total perceptualmotor system through each of the component sensorimotor systems. Directional linkages are shown set in a manner that would produce visual adaptation. (From Redding, Clark, \& Wallace, 1985.) 
prism exposure may be performed in the normal automatic manner, not requiring central processing capacity, and walking rate should be unrelated to either level of adaptation or performance of the secondary task. However, the continuous nature of optical flow is such that accurate locomotion can be maintained with occasional visual responses to the position ${ }^{3}$ of auditory or proprioceptive stimuli (cf. Thomson, 1983). That is, subjects can occasionally direct their eyes to look at, for example, another person speaking (i.e., the ear-eye linkage) or an obstacle they have just bumped into (i.e., the hand-eye linkage). On these occasions, discordance and adaptive recalibration occur in the responding visual system. The direction of linkage between systems can also be reversed, with consequent change in the locus of adaptation. For instance, when a subject reaches for a seen object, discordance and recalibration occur in the responding hand-head system (i.e., proprioceptive adaptation). Such directional linkage of visual and nonvisual systems requires central processing capacity, and when capacity is not available because it has been allocated to, for example, mental arithmetic, intersystem coordination is weakened and adaptation is reduced.

A further prediction from this model is that interference with adaptation should be graded and directly related to the difficulty of the secondary task. If the secondary task is relatively easy, drawing only infrequently on central processing capacity, some capacity should be available to occasionally link discordant systems. Consequently, level of adaptation should be higher under those conditions than under conditions in which greater demand is placed on central processing capacity by more difficult secondary tasks. The present experiment was designed to test this prediction. Three mental addition tasks of increasing difficulty were developed by varying the size of the correct arithmetic sum and the complexity of the required "carry" operations. Level of adaptation for the three experimental groups receiving the different levels of problem difficulty was compared with that of a control group which did not receive problems.

In other respects, the method was similar to previous studies (Redding et al., 1985) and conformed to the usual prism adaptation paradigm in which perceptual change is assessed as a difference score for criterion tests administered before and after prism exposure. The salient methodological features of these tests are the following: (1) Responses required by the tests are different from behavior required during exposure, and thus the tests are uncontaminated by any compensatory motor learning that may have occurred during exposure. (2) Tests are performed without prisms and there is no reason to expect contamination by any corrective rule consciously acquired during exposure. (3) The direction of change in test performance (pre- to postexposure) that would compensate for the distortion can be specified beforehand, and thus adaptive change can be identified. (4) Specific tests can be constructed which involve only a single perceptual system and thus are exclusively sensitive to different loci (kinds) of perceptual change. Three specific tests are commonly used in studies of adaptation to the optical transforms of displacement and tilt. These are tests that are sensitive to changes in (1) the visual system, (2) a proprioceptive system, or (3) both of these kinds of systems.

In studies of displacement adaptation like the present one, the interest is in change in egocentric direction. In visual tests, subjects indirectly adjust a target to appear straight ahead of the nose in an otherwise homogeneous field. This test is logically sensitive only to change in the eye-head system and the expected (adaptive) pre-post performance difference is in the direction of the displacement. A proprioceptive test requires a subject to point straight ahead of the nose with vision occluded.. This test is sensitive to change in proprioceptive systems (e.g., hand-to-head), and the adaptive direction is opposite to the displacement. A test for visual and/or proprioceptive adaptation requires a subject to point at a visual target with the unseen hand (i.e., open loop). This test is sensitive to any changes in the eye-to-hand systems, since it involves coordination of visual and proprioceptive systems and the adaptive direction is opposite to the displacement.

Visual and proprioceptive adaptation should be viewed as complementary rather than opposing processes. Both kinds of adaptation usually occur, in varying amounts, and each represents a part of the total adaptive response. In both tilt and displacement adaptation, the simple sum of visual and proprioceptive measures usually equals the total amount of adaptation, as measured by the open-loop eye-hand coordination test (e.g., Redding, 1978; Redding \& Wallace, 1976, 1978; Templeton, Howard, \& Wilkinson, 1974; Wallace, 1977; Wallace \& Redding, 1979; Welch, 1974; Welch, Choe, \& Heinrich, 1974; Wilkinson, 1971). Thus, adaptation seems to consist largely of local recalibration of perceptual inputs (Howard, 1971, 1982). Adaptive changes at higher levels, such as the coordinative mapping of perceptual inputs onto motor systems (e.g., Hardt, Held, \& Steinbach, 1971), would predict greater adaptive change in the eye-hand coordination test than the sum of visual and proprioceptive measures, because these latter tests are insensitive to changes between systems.

A perplexing variety of exposure conditions is known to produce different (but additive) amounts of one or the other kind of adaptation. For example, when a subject is required to walk about hallways wearing prisms (hall exposure), adaptation is more visual than proprioceptive, especially when sight of the body is restricted (e.g., Redding, 1978; Redding \& Wallace, 1976), but when a subject views his/her stationary feet (foot exposure), adaptation is entirely visual (Craske, 1967; Craske \& Crawshaw, 1974, 1978). On the other hand, if a subject is allowed a continuous view of his/her reaching hand while wearing prisms (concurrent exposure), adaptation tends to be more proprioceptive than visual (e.g., Harris, 1965; Kalil \& Freedman, 1966), but when sight of the hand is allowed only at the end of the reaching movement 
(terminal exposure), adaptation tends to be more visual than proprioceptive (e.g., Uhlarik \& Canon, 1971).

This variable, but additive, character of prism adaptation is one of the primary challenges for theories of adaptive systems, and we will generalize the directionalityof-guidance hypothesis to other exposure conditions in the following discussion. A primary tenet of our approach to the problem is that adaptation involves a central processor whose limited capacity to strategically control behavior can be allocated to one task only at the expense of a graded degradation in the performance of another task. Redding et al. (1985) established the idea of cognitive interference that seems to slow the adaptive process. The present experiment was a more definitive test of graded interference, using a secondary task that varied in level of difficulty and was performed simultaneously with the primary task of hall exploration.

\section{METHOD}

\section{Subjects}

The 60 subjects were right-handed undergraduate volunteers at Cleveland State University. All had normal or corrected-to-normal (contact lenses only) vision.

\section{Exposure Conditions}

All subjects walked back and forth along a short hall $(9.1 \mathrm{~m}$ long $\times 1.7 \mathrm{~m}$ wide) for $10 \mathrm{~min}$, wearing goggles that afforded a monocular (right eye) view through a Risley prism that laterally displaced the visual field $30 \mathrm{D}\left(17.1^{\circ}\right)$ in the rightward direction. Four groups of 15 subjects each received different levels of the secondary task.

Three groups received arithmetic problems designed to differ in difficulty. The simplest problems were single-digit problems (IDP) created by generating all the unordered pairs of the single digits from 1 to 9 , excluding identical pairs. These 36 single-digit problems were randomly ordered in repeated blocks prior to the experiment and were presented aloud to subjects in the form of addition questions (e.g., " $3+4=$ ?"). A more difficult problem set was created by generating all the unordered pairs of double digit numbers from 20 to 49 , also excluding identical pairs. These 435 doubledigit pairs were also randomly ordered and presented aloud as addition questions (e.g., " $47+39=$ ?"). Thus, this group (2DP2DA) received double-digit problems (2DP) whose answers were also only double digits (2DA). The third group received the most difficult task (e.g., "95 $+79=$ ?"), consisting of double-digit addition problems whose answers were triple digits (i.e., 2DP-3DA). The problem set for this group was the $\mathbf{4 3 5}$ randomly ordered pairs of the double digits from 70 to 99 , again excluding identical pairs and pairs that differed only in the ordinal position of the two doubledigit numbers (i.e., the set of unordered double-digit pairs). Subjects were required to solve as many problems as accurately and as quickly as possible while walking in the hall, and the accompanying experimenter recorded the number of problems attempted and the number correctly solved. The fourth (NP) group did not receive problems during hall exposure, and verbal interaction with the accompanying experimenter was discouraged.

As in previous experiments (Redding et al., 1985), subjects not receiving problems were encouraged to walk slowly while those receiving problems were encouraged to walk rapidly. This instruction was intended to counter the natural tendency for problem solving to slow walking rate and to control for the possibility that reduced adaptation might be attributed to a general reduction in exploratory behavior. "Finally, all subjects were instructed not to look at their hands or feet, and none were observed to violate this prohibition.

\section{Tests}

Before and after the 10-min hall exposure, the subjects were tested 10 times on each of three kinds of tests with the prism set to $0 \mathrm{D}$. The test for visual shift (VS) required the subjects to indicate verbally when a vertical line $(.2 \times 8 \mathrm{~cm}$ at a viewing distance of $60 \mathrm{~cm})$ appeared to be straight ahead of the nose when moved laterally across the visual field by the experimenter. Starting position of the test line was randomly varied, but was equally frequent in left and right visual fields. The test for proprioceptive shift (PS) required a subject to point straight ahead of the nose with the right hand when vision was occluded. A total shift (TS) measure of both VS and PS was obtained by having the subject point at the physically straightahead test line with his/her unseen right hand. For both the VS and TS tests, the visible field was illuminated but homogeneous, except for the target line. Level of adaptation was measured by the difference in degrees between average pretest and posttest performance, the adaptive direction of change being in the direction of the displacement for the VS test and opposite to the displacement for the PS and TS tests. (For additional details of test procedures and apparatus, see Wallace \& Redding, 1979.)

\section{Results}

Figure 2 illustrates the principal results. Proprioceptive shift was greater than visual shift $[\mathrm{F}(1,56)=47.74$, $\mathrm{p}<.001]$, but both kinds of adaptation suffered more interference as mental arithmetic increased in difficulty $[\mathrm{F}(3,56)=9.31, \mathrm{p}<.001]$, and there was no interaction between problem difficulty and type of adaptation $[\mathrm{F}(3,56)=1.61]$. That the problems were different in difficulty is demonstrated by the decreasing percentage of correctly solved problems over the three experimental groups $[F(2,42)=37.33, p<.001]$. The mean number of problems attempted $(234.3,92.1$, and 62.2 for 1DP, 2DP-2DA, and 2DP-3DA groups, respectively) reflected

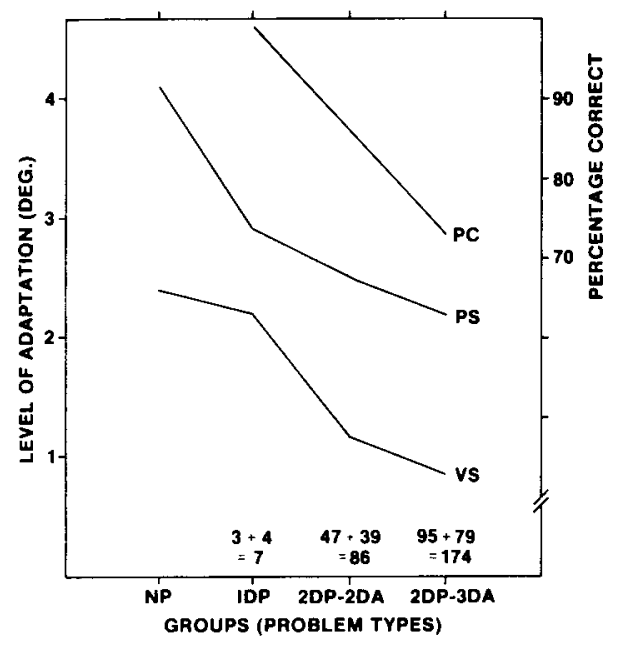

Figure 2. Cognitive interference in prism adaptation. Level of visual shift (VS) and proprioceptive shift (PS) and percentage of correctly solved mental addition problems (PC) as a function of four levels of problem difficulty: no problems (NP), single-digit problems (1DP), double-digit problems with double-digit answers (2DP-2DA), and double-digit problems with triple-digit answers (2DP-3DA). Examples of problem types are shown at appropriate points along the abscissa. 
the same increasing difficulty of problem types $[\mathrm{F}(2,42)$ $=296.86, \mathrm{p}<.001]$.

Data collected for the last five subjects tested in each group showed that walking rate tended to vary inversely with the difficulty of the cognitive task. A Newman-Keuls test $(p<.05)$ revealed that walking rates for 1DP $(58.4 \mathrm{~m} / \mathrm{min})$ and 2DP-2DA $(54.3 \mathrm{~m} / \mathrm{min})$ were not significantly different, but walking rate was slower for 2DP3DA $(45.4 \mathrm{~m} / \mathrm{min})$. More importantly, walking rate for the no-problem control group $(38.4 \mathrm{~m} / \mathrm{min})$ was slower than that for the slowest experimental group. To the extent that walking rate is an adequate index of exploratory behavior, this result supports the previous conclusion (Redding, Clark, \& Wallace, 1985) that reduced adaptation cannot be attributed to any general decrease in exploratory behavior.

Finally, it can be reported that there was no significant and little numerical difference between the TS test (4.74) and the sum VS + PS $(4.80)[F(1,56)<1.00]$. Neither did the differences among groups vary as a function of this factor $[F(3,56)=1.14]$. That is, the data reflect almost perfect additivity (e.g., Redding \& Wallace, 1976, 1978; Wallace, 1977; Wilkinson, 1971).

\section{DISCUSSION}

The fact that interference is graded, paralleling the cognitive difficulty of the interfering task, supports the idea that prism adaptation involves a limited-capacity centralprocessing mechanism, and it seems reasonable to suppose that central control processes are required to coordinate the discordant visual system with other sensorimotor systems. When central processing capacity is diverted to other tasks, such as mental arithmetic, the frequency of coordinated activity involving the discordant system is reduced with consequential reduction in intersystem discordance and adaptation.

The finding that PS was greater than VS contrasts sharply with the findings of previous studies (e.g., Redding, 1978; Redding et al, 1985; Redding \& Wallace, 1976), in which adaptation in hall exposure has usually been found to be largely, even exclusively, visual in nature. The directionality-of-guidance model (see Figure 1) suggests a possible reason for this difference. An obvious difference between studies is that previously the hallways included noisy, human traffic, whereas, for the present study, the area had been evacuated preparatory for renovation. Consequently, the frequency of subject encounters with visible sound sources was virtually eliminated for the present study. In the absence of auditory stimuli, the subjects may have responded more with proprioceptive anticipation of seen obstacles, thereby showing more proprioceptive adaptation. Thus, according to the model, adaptation should not occur if subjects are prevented from engaging in exploratory behavior by the provision of a fixation target for walking during hall exposure, but automatically guided locomotion should be facilitated by such restriction of information pickup to optical flow. The availability of sound sources and obstacles in the hall should enhance visual adaptation by providing occasions for nonvisual guidance of visual exploration, but if the nonvisual stimulus sources are first seen, they may produce visual guidance of nonvisual exploration (listening and touching) and should prompt nonvisual adaptation. Preliminary observations in our laboratories support these predictions, but further work remains to be done before a definitive statement can be made.

The directionality-of-guidance hypothesis is applicable to tasks other than hall exposure. Figure 3 illustrates the directionality-of-guidance model for eye-hand coordination exposure tasks. As shown in the figure, directional linkage is such as is presumed to occur in many concurrent exposure conditions. Visual information is used to guide the continuously visible hand, local discordance occurs between the remotely (visually) commanded hand position and the felt position of the hand necessary to achieve a target, and perceptual recalibration occurs in the proprioceptive system. On the other hand, the directional switch is largely reversed in terminal exposure conditions. Visual control of the nonvisible hand is largely precluded, but at the end of a reaching movement, corrective eye movements to fixate the optically displaced hand produce local discordance between the remotely (proprioceptively) commanded eye position and the visually encoded position of the hand, and perceptual recalibration occurs in the visual system. Thus, according to the model, experimental control over the directional linkage between discordant systems should produce predictable changes

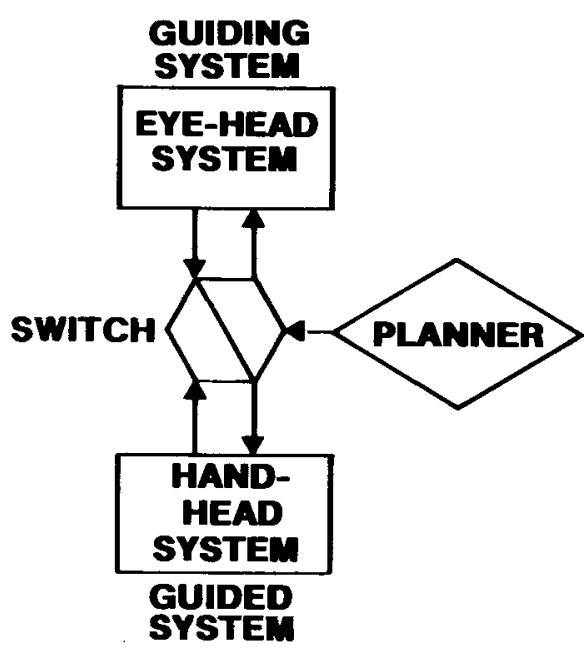

Figure 3. Directionality of guidance model for eye-hand coordination exposure tasks. Coordination requires directional guidance linkage between semi-autonomous eye-head (visual) and hand-head (proprioceptive) sensorimotor systems. When misalignment occurs (e.g., in the visual system), intentional control is necessary to establish and maintain appropriate intersystem linkage. Discordance occurs between the remote guidance signal and the local positional code, and recalibration occurs in the guided system. The linkage direc tion shown is such as would produce proprioceptive adaptation. 
in the locus of adaptation. Such coordinative linkage produces strategically appropriate linear organizations of component subsystems of the total perceptual-motor system, and local adaptive changes should be additive regardless of their relative magnitude. Total adaptation should be determined by the proportion of exposure activity spent with discordant systems linked. If central capacity is not allocated to link discordant systems, level of adaptation will be small or zero.

Finally, we should like to propose the directionalityof-guidance hypothesis as an alternative to the directedattention hypothesis, which assumes that adaptation occurs in the "nonattended" modality (e.g., Canon, 1970; Kelso, Cook, Olson, \& Epstein, 1975). We interpret "attended" as "guiding" and adopt the informationprocessing strategy of associating "attention" with the operation of a limited-capacity central processor. Whether there is a further meaning to the term is an empirical question. For example, it may be that the relative "weight" given to a modality in focal awareness is also a determinant of the locus of adaptation. ${ }^{5}$

Further research is clearly needed to understand the determinants of adaptation, but the present data encourage the hope that the directionality-of-guidance model will provide a framework for understanding the variable adaptive capacity of the perceptual-motor system. Explaining adaptation in such complicated naturalistic settings as hall exposure is one of the more difficult, but also more heuristic, challenges for theories of adaptive behavior, and we cannot hope to understand such behavior until we can explain the variable nature of adaptation in different eyehand coordination tasks.

\section{REFERENCES}

CANON, L. K. (1970). Intermodality inconsistency of input and directed attention as determinants of the nature of adaptation. Journal of Experimental Psychology, 84, 141-147.

CRASKE, B. (1967). Adaptation to prisms: Change in internally registered eye-position. British Journal of Psychology, 58, 329-335.

Craske, B., \& Crawshaw, M. (1974). Adaptive changes of opposite sign in the oculomotor systems of the two eyes. Quarterly Journal of Experimental Psychology, 26, 106-113.

Craske, B., \& Crawshaw, M. (1975). Oculomotor adaptation to prisms is not simply a muscle potentiation effect. Perception \& Psychophysics, 18, $105-106$.

Craske, B., Crawshaw, M. (1978). Spatial discordance is a sufficient condition for oculomotor adaptation to prisms: Eye muscle potentiation need not be a factor. Perception \& Psychophysics, 23, 75-79.

Ebenholtz, S. M. (1974). The possible role of eye-muscle potentiation in several forms of prism adaptation. Perception, 3, 477-485.

EBENHOLTZ, S. M. (1976). Additivity of aftereffects of maintained head and eye rotations: An alternative to recalibration. Perception \& Psychophysics, 19, 113-116.

Fitch, H. L., Tuller, B., \& Turvey, M. T. (1982). The Bernstein perspective: III. Tuning of coordinative structures with special reference to perception. In J. A. S. Kelso (Ed.), Human motor behavior. Hillsdale, N.J: Erlbaum.

HAMILTON, C. R. (1964). Studies of adaptation to deflection of the visual field in split-brain monkeys and man. Unpublished doctoral dissertation, California Institute of Technology.

Hardt, M. E., HeLd, R., \& Steinbach, M. J. (1971). Adaptation to displaced vision: A change in central control of sensorimotor coordination. Journal of Experimental Psychology, 89, 229-239.

HaRris, C. S. (1965). Perceptual adaptation to inverted, reversed, and displaced vision. Psychological Review, 72, 419-444.

HARRIS, C. S. (1980). Insight or out of sight? Two examples of perceptual plasticity in the human adult. In C. S. Harris (Ed.), Visual coding and adaptability. Hillsdale, NJ: Erlbaum.

Howard, I. P. (1971). Perceptual learning and adaptation. British Medical Bulletin, 27, 248-252.

HowARD, I. P. (1982). Human visual orientation. New York: Wiley.

Howard, I. P., \& Templeton, W. B. (1966). Human spatial orientation. New York: Wiley.

KALIL, R. E., FREEDMAN, S. J. (1966). Intermanual transfer of compensation for displaced vision. Perceptual and Motor Skills, 22 123-126.

Kelso, J. A. S., Cook, E., Olson, M. E., Epstein, W. (1975). Allocation of attention and the locus of adaptation to displaced vision. Journal of Experimental Psychology, 1, 237-245.

LEE, D. N., * Thomson, J. A. (1982). Vision in action: The control of locomotion. In D. J. Ingle, M. A. Goodale, \& R. J. W. Mansfield (Eds.), Analysis of visual behavior. Cambridge, MA: M.I.T. Press.

Longuet-Higgins, H. C., \& Prazdny, K. (1980). The interpretation of a moving retinal image. Proceedings of the Royal Society of London, Series B, 208, 385-397.

POSNER, M. I., \& SNYDER, C. R. R. (1975). Attention and cognitive control. In R. L. Solso (Ed.), Information processing and cognition. Hillsdale, NJ: Erlbaum.

REDDING, G. M. (1978). Additivity in adaptation to optical tilt. Journal of Experimental Psychology: Human Perception and Performance, 4, 178-190.

Redding, G. M., Clark, S. E., Wallace, B. (1985). Attention and prism adaptation. Cognitive Psychology, 17, 1-25.

Redding, G. M., \& Wallace, B. (1976). Components of displacement adaptation in acquisition and decay as a function of hand and hall exposure. Perception \& Psychophysics, 20, 453-459.

REDdING, G. M., \& WALLACE, B. (1978). Sources of "overadditivity" in prism adaptation. Perception \& Psychophysics, 24, 58-62.

SCHNEIDER, W., SHIFFrin, R. M. (1977). Automatic and controlled information processing in vision. In D. LaBerge \& S. J. Samuels (Eds.), Basic processes in reading. Hillsdale, NJ: Erlbaum.

SEGAL, S. J., \& FuSELLA, V. (1970). Influence of imagining pictures and sounds on detection of visual and auditory signals. Joumal of Experimental Psychology, 83, 458-464.

Templeton, W. B., Howard, I. P., \&ilkinson, D. A. (1974). Additivity of components of prismatic adaptation. Perception \& Psychophysics, 15, 249-257.

TномsoN, J. A. (1983). Is continuous visual monitoring necessary in visually guided locomotion? Joumal of Experimental Psychology: Human Perception and Performance, 9, 427-443.

Uhlarik, J. J., \& Canon, L. K. (1971). Influence of concurrent and terminal exposure conditions on the nature of perceptual adaptation. Joumal of Experimental Psychology, 91, 233-239.

WALLACE, B. (1977). Stability of Wilkinson's linear model of prism adaptation over time for various targets. Perception, 6, 145-151.

WALlace, B., \& Redding, G. M. (1979). Additivity in prism adaptation as manifested in intermanual and interocular transfer. Perception and Psychophysics, 25, 133-136.

WELCH, R. B. (1974). Speculations on a model of prism adaptation. Perception, 3, 451-460.

WelCh, R. B., ChOE, C. S., HeINRICh, D. R. (1974). Evidence for a three-component model of prism adaptation. Joumal of Experimental Psychology, 103, 700-705.

WiLkinson, D. A. (1971). Visual-motor control loop: A linear system? Joumal of Experimental Psychology, 89, 250-257.

\section{NOTES}

1. The term "visual adaptation" is used to designate adaptive change in the eye-head system which has phenomenal consequences for visual perception. The fundamental basis for such adaptation may be either 
a change in retinal local sign or in registered eye position (e.g., Craske \& Crawshaw, 1974; Harris, 1980). Current theory does not permit a test between these two possible accounts of visual change, and the present research is neutral with respect to this problem.

2. The precise effect of displacing prisms on optical flow is problematical, since the nature of the computational mechanisms which extract information from flow patterns is not completely understood (but see Longuet-Higgins \& Prazdny, 1980). Differential compression from prism base to apex may signal a "sideways" angle of approach, which may be responsible for the common tendency on the part of subjects to nonconsciously adopt a laterally turned head posture, thereby restoring normal flow patterns. Perhaps the best evidence that optical flow is not seriously distorted is the previously unreported observation that when subjects maintain fixation on a target at the end of the hall and make their turns with eyes closed, they can walk very rapidly and accurately and show no adaptation to the displacement.

3. The term "position"' is most readily interpreted to mean the perceptual dimension of location and its sensorimotor correspondents; however, a more generic meaning is also intended, including dimensions of orientation, size, and shape. Of course, not all such spatial channels are represented equally well in all sensorimotor systems (e.g., in the auditory system).

4. This manipulation was originally intended to equate walking rates for experimental and control groups, but it provides an even stronger control when it results in a faster walking rate for experimental groups.

5. Still another possibility is that residual muscle potentiation produced by asymmetric exercise of muscle groups is also a determinant of the locus of adaptation (e.g., Craske \& Crawshaw, 1975, 1978; Ebenholtz, 1974, 1976). Clear specification of alternative hypotheses and development of empirical tests is needed to determine the relative contributions of these various hypothetical mechanisms to the relative magnitude of local adaptive effects.

(Manuscript received September 25, 1984; Revisions accepted for publication January 30, 1985.) 